\title{
Systematic Computation of the Modal Spectrum of Boxed Microstrip, Finline, and Coplanar Waveguides Via an Efficient SDA
}

\author{
Gabriel Cano, Francisco Mesa, Member, IEEE, Francisco Medina, Member, IEEE, \\ and Manuel Horno, Member, IEEE
}

\begin{abstract}
This work reports an efficient and systematic procedure to obtain the complete modal spectrum of multilayer boxed planar lines. The complex propagation constants are obtained by computing the zeros of a properly built analytic complex function. This function is the product of two factors. One of them is the determinant function provided by the spectral domain-Galerkin analysis (SDA). The other factor is a function which cancels out the poles of the former factor without introducing additional poles nor zeros. The elimination of the poles overcomes numerical difficulties usually found in the zero searching process. In addition, powerful zero-searching integral techniques can be applied without problems. The numerical aspects involved in the computation of the spectral series are considered to speed up the computations. The features of an arbitrary number of propagating, evanescent, backward or complex modes of three important boxed structures (microstrip, finline, and coplanar waveguide) can be systematically studied with our method.
\end{abstract}

\section{INTRODUCTION}

$\mathbf{T}$ THE ANALYSIS OF discontinuities between boxed planar lines has been carried out by means of a wide variety of techniques. Among them, modal solution procedures are frequently chosen owing to a number of advantageous features discussed in the literature on this topic, e.g., [1]-[5]. In this context, microstrip, finline or CPW normal modes are likely the best choice as the basis for the modal expansion [6]. Nevertheless, tens of modes may be required to analyze a typical discontinuity [3], [5]. Consequently, it is convenient to develop a method to compute the propagation constants of an eventually large number of modes. The method should be reliable (avoiding missing of solutions), accurate (preserving mode orthogonality) and quick (saving CPU time). This paper tries to give a response to those requirements within the frame of the SDA.

The techniques used to analyze uniform planar lines usually lead to an homogeneous matrix equation that only can be solved for certain particular values (eigenvalues) of a parameter (the unknown propagation constant). These eigenvalues are complex solutions of a nonlinear equation which is obtained enforcing the determinant of the system matrix to vanish. Accuracy and reliability mainly depend on two factors: (1) accurate evaluation of the system matrix and (2) use of a

Manuscript received May 10, 1994; revised August 5, 1994. This work was supported by the CICYT, Spain, Project TIC91-1018.

The authors are with the Departamento de Electronica y Electromagnetismo, Facultad de Fisica, Universidad de Sevilla, 41012-Sevilla, Spain.

IEEE Log Number 9408578.

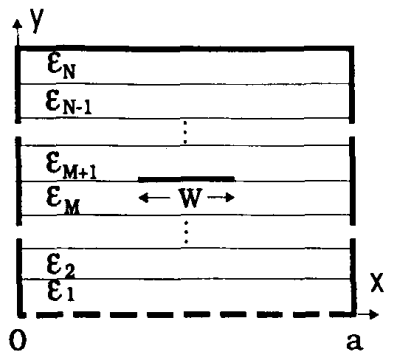

(a) (b)
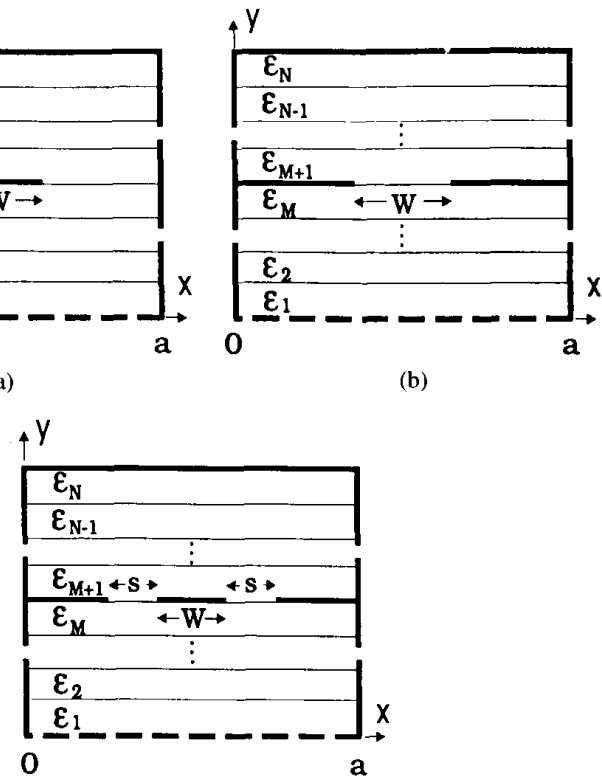

(c)

Fig. 1. Cross-section of the boxed lines in layered medium studied in this paper: (a) microstrip, (b) finline, and (c) coplanar waveguide (CPW). Dashed line stands for electric/magnetic wall. Anisotropic materials can be considered.

suitable method to solve the nonlinear dispersion equation. The numerical solution of this equation is difficult due to the existence of steep gradients and poles (often very close to the desired solution). In spite of its importance, only a few attempts of solving this problem can be found in the literature. In this way, the mode spectrum of finline [7], [8] and microstrip [4], [9] have been computed using the singular integral equation technique (SIE); the transverse resonance diffraction (TRD) method is applied in [10] to the unilateral finline. Particular interest deserves the space domain formulation reported in [11] to analyze a lossless single layer microstrip line. The zero-search problem is emphasized in those papers and treated using some a priori knowledge about pole location. Unfortunately, this is only feasible for particular situations. More recently, the singular value decomposition (SVD) method has been proposed in [12] -and used for the analysis of the quasi-TEM modes of multistrip structures in [13]- as a useful technique to relax the root location problem. 
This paper offers an alternative method presenting some advantages over the above mentioned procedures. We propose a general technique (a first step on which was reported in [14]) to deal with the computation of the modal spectrum of the generalized boxed planar lines in Fig. 1. Our method makes use of the well-established SDA formulation. Some authors sustain that SDA is suitable for the first few modes but not for higher order modes (e.g., [2], [3], [7], [8]). We have found that the possible drawbacks of the SDA are likely related to numerical inaccuracy when a relatively high number of basis functions is required and the lack of a suitable zero searching procedure.

We have developed a technique that improves accuracy and numerical stability, although the stress is here put on the development of a systematic strategy to find all the desired modes avoiding missing of solutions. This strategy is based on the cancellation of the poles of the characteristic dispersion equation. Thus, the dispersion relation is given in terms of the zeros of an analytic complex function (i.e., a function fulfilling the Cauchy-Riemman conditions). Note that, even though the method in [12] incorporates the important advantage of working with a function having no poles, that function is not analytic. On the contrary, our proposal takes advantage of the analyticity in the zero searching task. On the other hand, since our technique requires (such as many other methods) to evaluate the dispersion function many times, special attention has been paid to avoid redundant computation of the spectral series (most of the computing effort is concentrated in the first evaluation of the Galerkin matrix). This reduction of computation work and the aforementioned analyticity of the dispersion equation have been combined to implement a reliable, fast and systematic computer code for the analysis of the modal spectrum of the lines in Fig. 1.

\section{Problem Statement}

The analysis of the structures in Fig. 1 is conveniently carried out by means of the SDA. The spectral dyadic Green's function (SDGF), $\mathbf{G}\left(\alpha_{n}, \gamma, \omega\right)-\alpha_{n}$ is the Fourier variable, $\gamma=\beta-\jmath \alpha$, the unknown complex propagation constant and $\omega$ the angular frequency -, may be computed using the socalled EBM method [15], [16] (EBM allows us to deal with a complex linear layered medium). Once the SDGF is known, an integral equation is written either for the strip surface current (microstrip case) or for the slot electric field (finline and CPW cases). This spectral integral equation is solved via Galerkin's method, which leads to an homogeneous equations system. Its non trivial solution condition

$$
\operatorname{det}[\mathbf{A}(\gamma, \omega)]=0
$$

stands for the characteristic equation whose complex roots are the desired propagation constants $\left(\gamma_{i} ; i=1, \cdots, \infty\right)$. Two points concerning the solution of (1) should be now considered:

\section{A. Proper Computation of $[\mathbf{A}(\gamma, \omega)]$}

Computational efficiency is essential at this step of the analysis because it determines CPU time and accuracy. First of all we have to use adequate basis functions. We use first and second kind Chebyshev polynomials weighted with the Meixner edge condition [15], since this set of functions is specially suitable for the analysis of planar lines. Unfortunately, the spread spectrum of these functions yields very slowly convergent series. A huge number of Fourier terms could have to be retained to avoid serious truncation errors [17]. Moreover, the truncated spectral representation does not account for the edge singularity; therefore, the power of the weighted basis functions set can be only exploited if a suitable summation technique is provided. The problem becomes more noticeable for high order modes and very narrow strips/slots [18]. In this paper we use acceleration techniques that substantially relax all these inconveniences. Our technique has the additional virtue of avoiding redundant computations in the zero search process. A brief outline of the method can be found in the Appendix.

\section{B. Suitable Scheme for Complex Roots Searching}

The solution of (1) implies to seek for the zeros of a complex function of complex variable $F(\gamma, \omega) \equiv \operatorname{det}[\mathbf{A}(\gamma, \omega)]$, for each fixed frequency value. $F(\gamma, \omega)$ is not analytic but meromorphic. Its poles arise either from the poles of the SDGF (microstrip), or from the poles of its inverse (finline and $\mathrm{CPW}$ ). The presence of poles always makes difficult the zero searching task. Poles and zeros may be so close each other that numerical difficulties arise even if pole locations are known in advance, as in [11]. Therefore, we have developed an approach completely different from the one used in [7], [10]-[12]. Our strategy consists in obtaining the characteristic function of the nonstandard eigenvalue problem (1) in the form of a complex function which is analytic in the traced region. This function has smooth behavior so making easy the application of differential zero-searching algorithms (Muller or Davidenko's methods). However, we have exploited the advantages arising from the analyticity of that function since systematic integral methods based on well-known complex variable theorems can be then applied. We have implemented an improved version of the Delves-Lynnes [19] technique to obtain the number and location of all the zeros within a given region. The knowledge of the number of roots is useful so as to explore the complex plane systematically, so avoiding missing of solutions and redundancy. Although this technique gives very accurate results, additional accuracy is obtained-without increasing too much the CPU time-if these results are used as initial guesses of an iterative Muller algorithm.

\section{DISPERSION FUNCTION AS AN ANALYTIC COMPLEX FunCtion}

We have already discussed the advisability of formulating the dispersion problem in terms of the zeros of an analytic complex function. In this section we explain how to built this function. Let us begin with the microstrip configuration. In this case, (1) has the same poles than the SDGF. They are the propagation constants of the modes supported by the inhomogeneous waveguide remaining when the strip is removed, namely, the background waveguide (see Fig. 2(a)): only when 
the SDGF has a pole we can have tinite nonvanishing field in the absence of printed current. Although direct search for these poles is possible [11], this task could present difficulties when applied to a complex multilayer structure or when poles and zeros are in close proximity. So we have followed a different method that overcomes numerical problems. In [20] it was reported a procedure to compute the propagation constants of a laterally open waveguide with a bianisotropic layered medium in terms of the zeros of an unbounded analytic complex function. We follow here the guidelines contained in [20] but adapting the maths to account for the metallic enclosure so as to get an analytic characteristic equation for the eigenmodes of the background waveguide. Let $\tilde{\mathbf{E}}_{k}\left(\alpha_{n}\right)$ and $\tilde{\mathbf{J}}_{k}\left(\alpha_{n}\right)$ be the Fourier transforms of the tangential electric field and surface current at the $k$ th interface (a dependence on the Fourier variable $\alpha_{n}$ will be assumed henceforth for all the physical quantities). Using notation and formulas in [15] and [16] we can write two useful relationships between those quantities at the top, $N$, and bottom, 0 , interfaces (see Fig. 1)

$$
\begin{aligned}
\tilde{\mathbf{E}}_{N} & =\left[\boldsymbol{\Gamma}_{n}(\gamma, \omega)\right] \cdot \tilde{\mathbf{J}}_{0} ;(y=0 \text { e.w. }) \\
\tilde{\mathbf{E}}_{N} & =\left[\boldsymbol{\Gamma}_{n}(\gamma, \omega)\right] \cdot \tilde{\mathbf{E}}_{0} ;(y=0 \text { m.w. })
\end{aligned}
$$

(e.w. = electric wall; m.w. = magnetic wall), where

$$
\begin{array}{r}
{\left[\boldsymbol{\Gamma}_{n}\right]=\jmath \omega \mu_{0}[\boldsymbol{\Delta}]_{N, 1} \cdot[\mathbf{g}]_{0,1}^{-1} ; \quad(y=0 \text { e.w. })} \\
{\left[\boldsymbol{\Gamma}_{n}\right]=-\frac{1}{2}[\boldsymbol{\Delta}]_{N, 1} \cdot[\mathbf{g}]_{0,1}^{-1} \cdot[\mathbf{g}]_{0,0} ;(y=0 \text { m.w. })}
\end{array}
$$

and

$$
[\boldsymbol{\Delta}]_{N, 1}=(-1)^{N-1} \prod_{l=1}^{N-1}\left\{[\mathbf{g}]_{N-l, N-l+1}^{-1} \cdot[\mathbf{L}]_{N-l}\right\} .
$$

The $2 \times 2$ matrix functions $[\mathbf{L}]_{k}$ in (4) are computed using the following recurrence algorithm:

$$
\begin{gathered}
{[\mathbf{L}]_{k}=[\mathbf{g}]_{k, k}-[\mathbf{g}]_{k, k-1} \cdot[\mathbf{L}]_{k-1}^{-1} \cdot[\mathbf{g}]_{k-1, k}} \\
k=2, \cdots, N-1
\end{gathered}
$$

which has to be initialized with the expressions

$$
\begin{gathered}
{[\mathbf{L}]_{1}=[\mathbf{g}]_{1,1} ; \quad(y=0 \text { e.w. })} \\
{[\mathbf{L}]_{1}=[\mathbf{g}]_{1,1}-2[\mathbf{g}]_{1,0} \cdot[\mathbf{g}]_{0,0}^{-1} \cdot[\mathbf{g}]_{0,1} ; \quad(y=0 \text { m.w. }) .}
\end{gathered}
$$

The $2 \times 2$ matrix functions $[\mathbf{g}]_{i, j}$ in (3)-(6) are known in closed form and are given in [15] and [16]. It should be emphasized that the $2 \times 2\left[\boldsymbol{\Gamma}_{n}\right]$ matrices in (2) and the SDGF are simultaneously computed. Therefore, the evaluation of $\left[\boldsymbol{\Gamma}_{n}\right]$ requires negligible additional computation.

Now, the presence of the upper conducting plate is taken into account by imposing $\tilde{\mathbf{E}}_{N}=0$. Then, the nontrivial solution condition for $(2 a)$ or $(2 b)$

$$
\operatorname{det}\left[\boldsymbol{\Gamma}_{n}(\gamma, \omega)\right]=0 \quad(n=0,1,2, \ldots)
$$

gives the propagation constants of the modes in the inhomogeneous background waveguide. For our purpose, an important feature of (7) is that $\operatorname{det}\left[\Gamma_{n}(\gamma, \omega)\right]$ does not have any poles

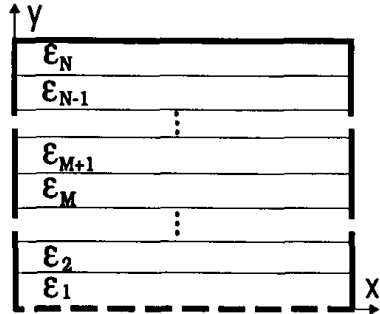

(a)

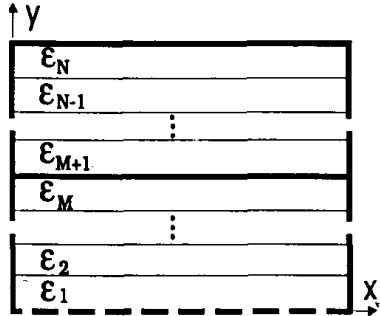

(b)
Fig. 2. Structures whose propagation constants are the poles of the dispersion characteristic equation (a) for the microstrip line and (b) for finline and CPW.

in the $\gamma$ complex plane [20]. We will take advantage of this property later but, before going on, let us discuss the finline/CPW case. For any of these structures, the kernel of the integral equation for the slot electric field is the inverse of the SDGF, i.e., $\left[\overline{\mathbf{G}}\left(\alpha_{n}, \gamma, \omega\right)\right]^{-1}$. The singularities in (1) now arise from the modes corresponding to the two waveguides resulting from the substitution of the printed $M$ th interface by a continuous conducting plate (see Fig. 2(b)). We can write for the lower $(L)$ and upper $(U)$ waveguides

$$
\begin{gathered}
\tilde{\mathbf{E}}_{M}=\left[\boldsymbol{\Gamma}_{n}^{L}\right] \cdot \tilde{\mathbf{J}}_{0} \quad \text { or } \quad \tilde{\mathbf{E}}_{M}=\left[\boldsymbol{\Gamma}_{n}^{L}\right] \cdot \tilde{\mathbf{E}}_{0} \\
\tilde{\mathbf{E}}_{N}=\left[\boldsymbol{\Gamma}_{n}^{U}\right] \cdot \tilde{\mathbf{J}}_{M}
\end{gathered}
$$

where $\left[\boldsymbol{\Gamma}_{n}^{L}\right]$ and $\left[\boldsymbol{\Gamma}_{n}^{U}\right]$ are obtained by following the same steps reported for the microstrip case. If we redefine

$$
\left[\boldsymbol{\Gamma}_{n}(\gamma, \omega)\right]=\left[\boldsymbol{\Gamma}_{n}^{U}\right] \cdot\left[\boldsymbol{\Gamma}_{n}^{L}\right]
$$

condition (7) still determines the propagation constants in this case.

The doubly infinite set of solutions of (7) is also the set of poles of the determinant function (1). In other words, we have built up a family of functions

$$
\left\{F_{n}(\gamma, \omega) \equiv \operatorname{det}\left[\boldsymbol{\Gamma}_{n}(\gamma, \omega)\right]\right\}_{n=0,1,2, \ldots}
$$

whose zeros are the poles of $F(\gamma, \omega) \equiv \operatorname{det}[\mathbf{A}(\gamma ; \omega)]$. These functions can be now used to eliminate the poles of $F(\gamma, \omega)$ without introducing additional poles or roots. Thus, the dispersion relation for the boxed transmission line-which was initially given in terms of the meromorphic expression (1) - is now more conveniently expressed by means of the following analytic characteristic equation:

$$
\operatorname{det}[\mathbf{A}(\gamma, \omega)] \cdot \prod_{n=0}^{\infty} F_{n}(\gamma, \omega)=0
$$

where, as will be shown later, just a few factors of the infinite product are required in practice.

\section{SYSTEMATIC SEARCH FOR THE ZERO'S}

Equation (9) turns out to be a rather formal expression, since infinite factors should be retained so as to built an analytic function in the entire complex plane (except at infinity). However, (9) has to be analytic only in that region of the complex plane to be traced by the zero-searching algorithm. 
Therefore only a few values of $n$ are usually required to generate a function

$$
H(\gamma, \omega) \equiv \operatorname{det}[\mathbf{A}(\gamma \cdot \omega)] \cdot \prod_{n=0}^{N_{\nu}} F_{n}(\gamma \cdot \omega)
$$

which is analytic in the region of interest. $N_{\nu}$ in $(10)$ is chosen in such a way that, for a given operation frequency, $\omega$, and box width, $a$. all the poles in the search region are removed (i.e., $\left|\beta^{2}\right|<\left(N_{\nu} \pi / a\right)^{2}-\epsilon_{r} k_{0}^{2}$, where $\epsilon_{r}$ stands for the modulus of the highest relative dielectric constant [7]).

Since $H(\gamma, \omega)$ is analytic in the working region, $R$, of the complex plane, the integral method refereed as C) in [19] can be used to search for its zeros. We have chosen this method instead of B) in [19] because it has been found that the numerical evaluation of the derivative of $H(\gamma, \omega)$ yields much better performance than the scheme proposed in B). This technique allows systematic exploration of the region $R$ in order to detect the number of zeros in it. We have found this method better than other alternatives, specially for regions with several and/or very close roots. Complex plane integrations are carried out along circular paths by means of high order Gauss-Legendre quadratures (20-40 points). This means that $H(\gamma, \omega)$ has to be evaluated many times before attaining the solution. Nevertheless, since several zeros may be computed with each set of values of $H(\gamma, \omega)$, the total number of evaluations of this function might be even smaller than those required when iterative methods are used. Thus, with similar computational effort, one gets more reliability and systematicity in the evaluation of the propagation constants In addition, thanks to the use of the asymptotic techniques commented in the Appendix, the main part of the numerical effort is concentrated in the first evaluation of $H(\gamma, \omega)$ : further evaluations do not increase too much the overall CPU time.

\section{DISCUSSION AND RESULTS}

The theory reported in previous sections is the basis for a set of computer Fortran codes that analyze the eigenmode spectrum of the structures in Fig. 1. (they include a variety of versions of the microstrip, finline, and CPW, including symmetrically coupled geometries and anisotropic uni- and biaxial substrates). Several aspects of the convergence and general performance of the technique have been exhaustively investigated (some comments about this are in the Appendix). However, in this paper we are more interested in posing and solving an adequate dispersion equation than in the numerical details involved in the generation of the Galerkin matrix (which are left for a next paper), although it is obvious that the latter problem had to be properly solved before going on. We want only to say than a highly accurate and efficient code has been developed which is able to deal with many basis functions while keeping numerical stability.

In Fig. 3 we have plotted the standard meromorphic characteristic function in (1), $F(\gamma, \omega)$, and the modified analytic characteristic function in $(10), H(\gamma, \omega)$ for a range of values of $\left(\beta / k_{i}\right)^{2}$. For simplicity, we have restricted the picture to the real axis (where the functions are real). Nevertheless, the conclusions obtained from its examination also apply to the

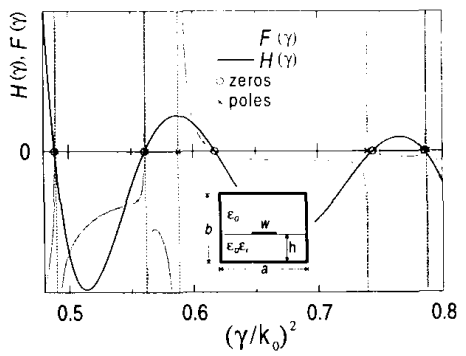

Fig. 3. Typical plots of $F(;, \omega)$ (meromorphic), $H(;, \omega)$ (analytic) dispersion functions. These plots correspond to a centered boxed microstrip on isotropic substrate $(c,=8.875)$ having the following dimensions: $h=1.27$ $\mathrm{mm}, a=b=12.7 \mathrm{~mm}$, and Freq. $=28 \mathrm{GHz}$ (this structure has been analyzed in $[11]$ ).

complex case. Fig. 3 shows how both functions have the same zeros, but $F(\gamma, \omega)$ has also poles, some of which are extremely close to the zeros (they could even match). This latter fact seriously complicates - or even precludes - the root identification with conventional methods, whereas does not affect our formulation.

Our results have been checked against many published data computed by means of SDA and other techniques. We have found excellent agreement with most of these results within the accuracy of usual graphical representations. However, we have found that missing of modes is very common. For instance, in Fig. 4 we plot the propagating and a few evanescent modes of a unilateral finline. Modes having odd ( $E$-modes) and even ( $M$-modes) symmetry with respect to the middle plane normal to the fin interface have been separated. The figure shows our results and results from [2] and [8]. This figure is interesting because the authors in [8] express an opinion about the suitability of the SDA to account for higher order modes: although SDA is a useful and well-founded method to deal with planar structures, it is not adequate for reliable and quick computation of higher order modes; SIE technique would be a better alternative. This opinion has been sustained by several authors elsewhere [2], [3], [7], [8]. This conclusion is supported in [8] by comparing SIE data with the results in [2] (SDA) for a finline structure. Following these authors, missing of solutions and relatively high computational cost would be drawbacks associated to SDA. However, Fig. 4 clearly shows that we find all the modes reported in [8] and a few more not reported in that paper (including one complex $M$-type mode). In addition, the use of the asymptotic techniques commented in the Appendix makes the SDA a very efficient method from the perspective of computational cost (of the same order than the very efficient SIE technique). An illustrative comparison has been carried out against the numerical values reported by Omar et al. [7] (SIE) for a large number of $E$-modes of a unilateral finline. Table I shows our data and the data in that paper. Apart from excellent agreement, it can be seen that we find three modes not reported in [7]. The lost modes are some of those having propagation constants very close to waveguide modes (singularities in the conventional formulation), as shown at the bottom of Table I. This closeness is likely the reason for missing of solutions in [7] (since the analytical/numerical analysis seems to be very careful). This 


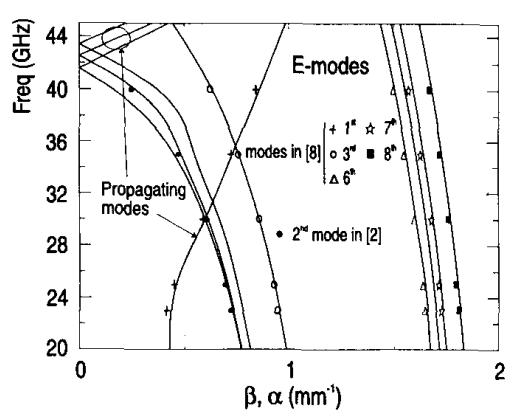

(a)

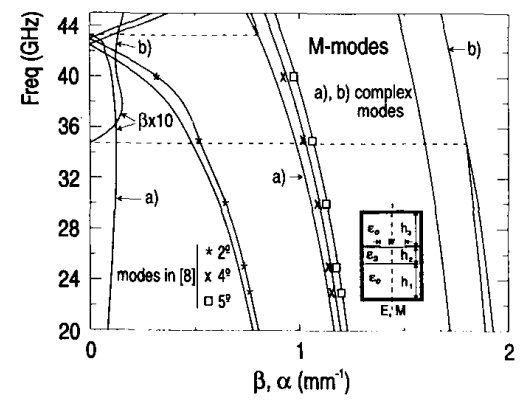

(b)

Fig. 4. Dispersion curves of several modes of a unilateral finline in WR-28 waveguide ( $\beta$ for propagating modes and $\alpha$ for evanescent ones). $\varepsilon_{r 1}=\varepsilon_{r 3}=1 ; h_{1}=h_{3}=3.429 \mathrm{~mm} ; \varepsilon_{r 2}=2.22 ; h_{2}=0.254 \mathrm{~mm} ; w=$ $1 \mathrm{~mm}$. (a) $E$-modes, i.e., modes having an electric wall in the middle plane; (b) $M$-modes, i.e., those having a magnetic wall. Solid lines: our results; symbols: data in [2] and [8].

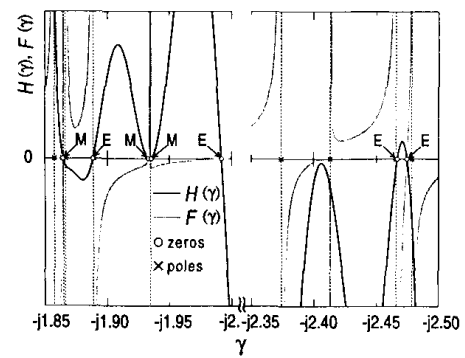

Fig. 5. Plot of $F(\gamma)$ (gray line) and $H(\gamma)$ (black line) for the structure whose modes are given in Table I. Even and odd symmetries with respect to the middle plane of each solution is noted.

conclusion is confirmed in Fig. 5. This figure shows both the meromorphic and the analytic dispersion functions for the case considered in Table I: Numerical problems associated to the use of the meromorphic function are clearly expected.

We have also made similar comparisons for the microstrip line analyzed in [4] with SIE. Some numerical data reported in that paper are compared with our data in Table II. Once again agreement is very good, but we find some modes not reported in [4] at $20 \mathrm{GHz}$. Fig. 6 shows the dispersion behavior of the modes in Table II. Circles correspond to the propagation constants not reported in [4]: It is clear that they are not spurious solutions. In our opinion, Tables I and II and Figs. 4-6 illustrate that, if properly implemented, SDA is a very
TABLE I

Propagation Constants of the E-Modes of the Unilateral. Finline Studied in [1]

\begin{tabular}{|c|c|c|c|c|c|c|c|}
\hline$\beta$ & 1 & 2 & 3 & 4 & 5 & 6 & 7 \\
\hline (a) & 0.6824 & $-j 0.6063$ & $-j 0.7444$ & $-j 1.5953$ & $-j 1.6487$ & $-j 1.6798$ & $-j 1.7468$ \\
\hline (b) & 0.6820 & $-j 0.6067$ & $-j 0.7448$ & $-j 1.5955$ & $-j 1.6489$ & $-j 1.6800$ & $-j 1.7440$ \\
\hline \hline$\beta$ & $8\left(^{*}\right)$ & 9 & $10\left(^{*}\right)$ & 11 & 12 & 13 & 14 \\
\hline (a) & $-j 1.888$ & $-j 1.9918$ & $-j 2.466$ & $-j 2.4746$ & $-j 2.5486$ & $-j 2.7066$ & $-j 3.0732$ \\
\hline (b) & - & $-j 1.9922$ & - & $-j 2.4747$ & $-j 2.5491$ & $-j 2.7070$ & $-j 3.0735$ \\
\hline \hline$\beta$ & 15 & $16\left(^{*}\right)$ & 17 & 18 & 19 & 20 & $21\left(^{*}\right)$ \\
\hline (a) & $-j 3.1135$ & $-j 3.2064$ & $-j 3.2370$ & $-j 3.3646$ & $-j 3.4763$ & $-j 3.4967$ & $-j 3.5961$ \\
\hline (b) & $-j 3.1136$ & $-j 3.2065$ & $-j 3.2372$ & $-j 3.3648$ & $-j 3.4763$ & $-j 3.4969$ & \\
\hline \hline$\beta$ & 22 & 23 & 24 & $25\left(^{*}\right)$ & 26 & 27 & 28 \\
\hline (a) & $-j 3.6056$ & $-j 3.6641$ & $-j 3.8124$ & $-j 3.8786$ & $-j 3.9307$ & $-j 3.9654$ & $-j 4.0196$ \\
\hline (b) & $-j 3.6057$ & $-j 3.6648$ & $-j 3.8127$ & $-j 3.8787$ & $-j 3.9308$ & $-j 3.9658$ & $-j 4.0196$ \\
\hline \hline$\beta$ & 29 & 30 & 31 & 32 & 33 & & \\
\hline (a) & $-j 4.0760$ & $-j 4.2311$ & $-j 4.3468$ & $-j 4.3637$ & $-j 4.4325$ & & \\
\hline (b) & $-j 4.0770$ & $-j 4.2314$ & $-j 4.3475$ & $-j 4.3637$ & $-j 4.4326$ & & \\
\hline
\end{tabular}

\begin{tabular}{|l|l|l|l|l|l|l|}
\hline Finline (*) & $-j 1.888412$ & $-j 2.466629$ & $-j 3.206430$ & $-j 3.596131$ & $-j 3.878616$ \\
\hline
\end{tabular}

\begin{tabular}{|c|c|c|c|c|c|}
\hline Waveguide & $-j 1.888413$ & $-j 2.466633$ & $-j 3.206437$ & $-j 3.596134$ & $-j 3.878553$ \\
$n=2$ & $n=2$ & $n=2$ & $n=4$ & $n=2$ \\
\hline
\end{tabular}

WR-28 Waveguide: $\varepsilon_{r 1}=\varepsilon_{r 3}=1 ; h_{1}=h_{3}=3.429 \mathrm{~mm} ; \varepsilon_{r 2}=2.22 ; h_{2}=$ $0.254 \mathrm{~mm} ; w=0.2 \mathrm{~mm}$; frequency $=30 \mathrm{GHz}$. (a) Our results. (b) Results in [1]. (*) Propagation constants very close to waveguide modes (see also Fig. 5) such as shown in the bottom part of the table ( $n$ is the order of the spatial hamonic in (7)).

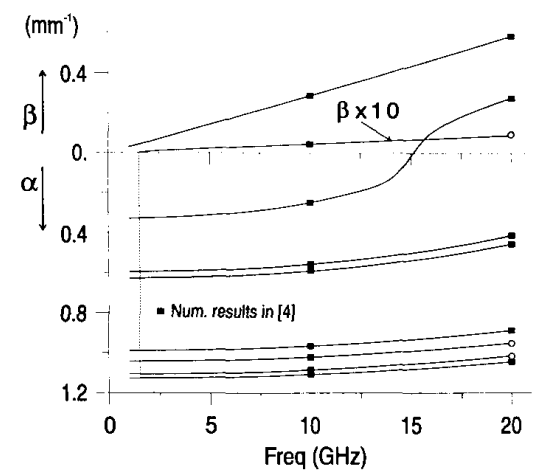

Fig. 6. Dispersion curves for the boxed microstrip ( $w=0.953 \mathrm{~mm}$ ) whose first few modes (at 10 and $20 \mathrm{GHz}$ ) are given in Table II. Circles represent modes at $20 \mathrm{GHz}$ not reported in [4] (one evanescent mode and one complex mode).

powerful tool for the analysis of planar structures also when very high order modes are traced. More examples and an exhaustive study of the performance of our formulation-in contrast with the conventional one-for a wide range of situations (with particular emphasis on the identification of complex modes) can be found in [21].

We would like to finish this section with an example of CPW structure. We have chosen one of the multilayer configurations proposed by Liu and Itoh in [22] to avoid leakage in conductor backed coplanar waveguides. Fig. 7 shows the mode spectrum for that structure when enclosed in a rectangular waveguide.

\section{CONCLUSION}

In this paper we have proposed a technique to overcome some difficulties arising when the conventional SDA is used 
TABLE II

Propagation Constants, i3, of a Boxed Microstrip [4]

\begin{tabular}{|c|c|c|c|c|}
\hline \multicolumn{5}{|c|}{ Frequency $=10 \mathrm{GHz}$} \\
\hline & \multicolumn{2}{|c|}{$\begin{array}{c}W=0.953 \mathrm{~mm} \\
\beta\left(\mathrm{mm}^{-1}\right)\end{array}$} & \multicolumn{2}{|c|}{$\begin{array}{c}W=4.572 \mathrm{~mm} \\
\beta\left(\mathrm{mm}^{-1}\right)\end{array}$} \\
\hline$n^{n}$ & (a) & (b) & (a) & (b) \\
\hline I & 0.2885 & 0.2884 & 0.3023 & 0.3021 \\
\hline & $-j 0.2484$ & $-j 0.2$ & $-j 0.2470$ & \\
\hline & $-j 0.5547$ & & & \\
\hline & $-j 0.5887$ & & $-j 0.6$ & \\
\hline & $-j 0.965$ & & & \\
\hline & $-j 1.0208$ & $-j 1.0$ & $-j 0.9$ & \\
\hline & $\mp 0.00450$ & & $-j 1 . c$ & \\
\hline & $\mp j 1.0852$ & $\mp j 1$ & $-j 1.1$ & \\
\hline & $j 1.1078$ & $-j 1.1075$ & $-j 1.1237$ & \\
\hline \multicolumn{5}{|c|}{ Frequency $=$} \\
\hline & \multicolumn{2}{|c|}{$\begin{array}{c}W=0.953 \mathrm{~mm} \\
\beta\left(\mathrm{mm}^{-1}\right)\end{array}$} & \multicolumn{2}{|c|}{$\begin{array}{c}W=4.572 \mathrm{~mm} \\
\quad \beta\left(\mathrm{mm}^{-1}\right)\end{array}$} \\
\hline & (a) & (b) & (a) & (b) \\
\hline & 0.5828 & & 0.6136 & \\
\hline & 0.2781 & & 0.27 & \\
\hline & $-j 0.4126$ & $-j 0.4127$ & $-j 0.4033$ & \\
\hline & & & & \\
\hline & $-j 0.8884$ & $-j 0.8$ & $-j 0.8$ & \\
\hline & $-j 0.9512$ & - & $-j 0.8989$ & \\
\hline & & & & \\
\hline & 0156 & -- & $-j 1.0391$ & \\
\hline & & & & \\
\hline
\end{tabular}

Dimensions: $a=9.52 \mathrm{~mm}: h_{1}=0.635 \mathrm{~mm} ; \varepsilon_{r 1}=2.32 ; h_{2}=5.715 \mathrm{~mm} ; \varepsilon_{r^{2}}=$ 1. (a) Our results. (b) Results in [4]. Modes 7 and 8 for $w=0.953 \mathrm{~mm}$ are the real (7) and imaginary (8) parts of a pair of complex modes.

to calculate the propagations constants of higher order modes of planar transmission lines. The limitation of the SDA to the computation of the first few modes has been removed by means of two concurrent improvements: (1) drastic enhancement of the numerical performance of SDA through a suitable preprocessing of the spectral series and (2) formulation of the dispersion equation in terms of an analytic complex characteristic function. The final result is a set of quick, accurate and reliable computer codes to obtain the complete modal spectrum of the structures shown in Fig. 1. These programs are potentially useful for the modal analysis of discontinuities in those structures.

\section{APPENDIX}

\section{A. Numerical Treatment of the Galerkin Matrix}

Each entry of the SDA Galerkin's matrix, $[\mathbf{A}(\gamma, \omega)]$, is an infinite, slowly convergent series involving the product of the Fourier transforms of two basis functions and the appropriate element of the SDGF. Drastic acceleration is obtained by extracting out and analytically adding their asymptotic limit behavior [23]. With our choice of basis functions all the elements of $[\mathbf{A}]$ have the same asymptotic functional limit, $A_{p, q}^{\text {as }}$, so we only have to deal with series of the following type:

$$
A_{p, q}^{\text {as }}=\sum_{n=1}^{\infty} J_{p}(\alpha n) J_{q}(\alpha n) \quad\left\{\begin{array}{l}
\sin (c n) \\
\cos (c n)
\end{array}\right\} \mathcal{F}^{\text {as }}(n, \omega, \gamma)
$$

where $\mathcal{F}^{\text {as }}(n, \omega, \gamma)$ is an asymptotic simplified approximation of an element of the SDGF (or related quantity) and $J_{p}$ stands

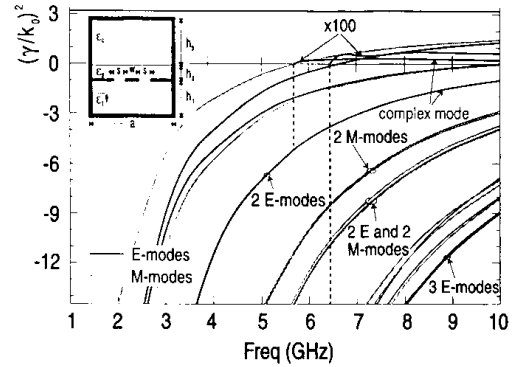

Fig. 7. Dispersion curves for a number of modes of a boxed multilayer CPW structure. Dimensions: $a=15 \mathrm{~mm} ; h_{1}=0.635 \mathrm{~mm} ; z_{r 1}=2.33 ; h_{2}=0.06$ $\mathrm{mm} ; \bar{s}_{r_{2}}=10.5 ; h_{3}=15 \mathrm{~mm} ; s_{3}=1 ; w=s=0.254 \mathrm{~mm}$.

for the first kind Bessel function of integer order $p . \alpha$ and $c$ are functions of the ratio between the strip (or slot) width and the box width. The difference between the actual series and its asymptotic expression vanishes very quickly if $\mathcal{F}^{\text {as }}$ is properly defined. In our case we obtain $1 / n^{5}$ behavior by using:

$$
\mathcal{F}^{\mathrm{as}}=\frac{g_{1}(\omega \cdot \gamma)}{n}+\frac{g_{3}(\omega, \gamma)}{n^{3}}
$$

where $g_{1,3}$ are known in closed form [21] (also in [23] for the microstrip case including the special feature of very thin layers).

Our problem reduces to compute $A_{p, q}^{\text {as }}$ in (A1) very efficiently. The part of $A_{p, q}^{\text {as }}$ corresponding to the first second hand term in (A2) is associated to the quasistatic limit of the problem, and it has been quasianalytically computed in [24] and [25]. The method in [24] and [25] consists in transforming the numerical series in a convolution/inner product integral in the spatial domain which is almost analytically evaluated. For the part involving the factor $1 / n^{3}$ we have used a similar idea [21]. This method is better than the one used in [23] while still keeping an important feature also mentioned in [23]: the asymptotic part of the computations is carried out just once for a given structure (a similar property was also used in [11]). This fact is very important because the root searching task requires multiple evaluations of the Galerkin matrix. The final result from all this algebra is that the number of spectral terms to be retained in series summation is drastically reduced: no more than a few units or tens of spectral terms are necessary for microstrip calculations (fewer terms are typically required for finline and CPW). Note that thousands of terms may be required if the asymptotic method is not applied (see, for instance, [17]). This makes the efficiency of SDA similar to SIE while keeping the generality of SDA. In the context of this work, there is another reason for doing this analytical processing of the spectral series apart from computational speed: the reaction terms in the Galerkin matrix involving high order Chebyshev polynomials have to be accurately computed when very high order modes are treated in order to keep mode orthogonality. We have not either detected spurious solutions with our method. For a more detailed study of the technique sketched in this Appendix, the reader is referred to $[21]$. 


\section{REFERENCES}

[1] A. S. Omar and K. Schünemann, "Transmission matrix representation of finline discontinuities," IEEE Trans. Microwave Theory Tech., vol. MTT-33, pp. 765-770, Sept. 1985.

[2] M. Helard, J. Citerne, O. Picon, and V. F. Hanna, "Theoretical and experimental investigation of finline discontinuities," IEEE Trans. Microwave Theory Tech., vol. MTT-33, pp. 994-1003, Oct. 1985.

[3] Q. Xu, K. J. Webb, and R. Mittra, "Study of modal solution procedures for microstrip step discontinuities," IEEE Trans. Microwave Theory Tech., vol. 37, pp. 381-387, Feb. 1989.

[4] N. K. Uzunoglu, C. N. Capsalis, and C. P. Chronopoulos, "Frequencydependent analysis of a shielded microstrip step discontinuity using an efficient mode-matching technique," IEEE Trans. Microwave Theory Tech., vol. MTT-36, pp. 976-984, June 1988.

[5] C. J. Railton and T. Rozzi, "The rigorous analysis of cascaded step discontinuities in microstrip," IEEE Trans. Microwave Theory Tech., vol. MTT-36, pp. 1177-1185, July 1988.

[6] A. S. Omar and K. F. Schünemann, "The effects of complex modes at finline discontinuities," IEEE Trans. Microwave Theory Tech., vol. MTT-34, pp. 1508-1514, Dec. 1986.

[7] ___ "Formulation of the singular integral equation technique for planar transmission lines," IEEE Trans. Microwave Theory Tech., vol. MTT-33, pp. 1313-1322, Dec. 1985.

[8] Y.-F. Huang and S.-L. Lai, "Regular solution of singular integral equation for finline," Inst. Elect. Eng. Proc., vol. 140, pt. H, no. 1, pp. 11-16, Feb. 1993.

[9] W. X. Huang and T. Itoh, "Complex modes in lossless shielded microstrip lines," IEEE Trans. Microwave Theory Tech., vol. MTT-36, pp. 163-165, Jan. 1988

[10] C. A. Olley and T. E. Rozzi, "Systematic characterization of the spectrum of unilateral finline," IEEE Trans. Microwave Theory Tech., vol. MTT-34, pp. 1147-1156, Nov. 1986

[11] C. J. Railton and T. Rozzi, "Complex modes in boxed microstrip," IEEE Trans. Microwave Theory Tech., vol. MTT-36, pp. 865-874, May 1988.

[12] V. A. Labay and J. Bornemann, "Matrix singular value decomposition for pole-free solutions of homogeneous matrix equations as applied to numerical modeling methods," IEEE Microwave and Guided Wave Lett. vol. 2, pp. 49-51, Feb. 1992.

[13] M. Borge, A. Casanueva, and J. L. García, "Efficient eigenmode analysis for multiconductor and multilayer planar transmission lines: SVD a technique capable of solving homogeneous matrix equations without poles," in Proc. 23rd Eur. Microwave Conf., Madrid, Spain, Sept. 1993, pp. 531-534.

[14] G. Cano, F. Mesa, F. Medina, R. Marqués, and M. Horno, "Estudio sistemático de los modos de propagación en líneas planares encapsuladas," (in Spanish) in VI Symp. U.R.S.I., Cáceres, Spain, Sept. 1991, pp. 215-218.

[15] F. Medina, M. Horno, and H. Baudrand, "Generalized spectral analysis of planar lines on layered media including uniaxial and biaxial dielectric substrates," IEEE Trans. Microwave Theory Tech., vol. 37, pp. 504-511, Mar. 1989.

[16] F. Mesa, R. Marqués, and M. Horno, "A general algorithm for computing the bidimensional spectral green's dyad in multilayered complex bianisotropic media: The Equivalent Boundary Method (EBM)," IEEE Trans. Microwave Theory Tech., vol. 39, pp. 1640-1649, Sept. 1991.

[17] J. T. Kuo and C. C. Tzuang, "Complex modes in shielded suspended coupled microstrip lines," IEEE Trans. Microwave Theory Tech., vol. 38, pp. 1278-1286, Sept. 1990.

[18] S. El-Gazhaly and W. J. R. Hoefer, "Effects of number of spectral terms on convergence of spectral-domain calculation of $E$-plane structures," Electron. Lett., vol. 21, no. 16, pp. 677-678, Aug. 1985.

[19] L. M. Delves and J. N. Lynnes, "A numerical method for locating the zeros of an analytic function," Math. Comp., vol. 21, pp. 543-560, 1967.

[20] R. Marqués, F. Mesa, and M. Horno, "Nonreciprocal and reciprocal complex and backward waves in parallel plate waveguides loaded with a ferrite slab arbitrarily magnetized," IEEE Trans. Microwave Theory Tech., vol. 41, pp. 1404-1418, Aug. 1993.

[21] G. Cano, "Frequency domain analysis of boxed planar structures in stratified medium," $\mathrm{Ph}$. D. dissertation, University of Seville, Spain, Sept. 1993 (in Spanish).

[22] Y. Liu and T. Itoh, "Leakage phenomena inmultilayered conductorbacked coplanar waveguides," IEEE Microwave Guided Wave Lett., vol. 3, pp. $426-427$, Nov. 1993 .

[23] G. Cano, F. Medina, and M. Horno, "Efficient spectral domain analysis of generalizaed multistrip lines in stratified media including thin, anisotropic and lossy substrates," IEEE Trans. Microwave Theory Tech., vol. 40, pp. 217-227, Feb. 1992.
[24] F. Medina and M. Horno, "Quasianalytical static solution of the boxed microstrip line embedded in a layered medium," IEEE Trans. Microwave Theory Tech., vol. 40, pp. 1748-1756, Sept. 1992.

[25] E. Drake, F. Medina, and M. Horno, "Quasianalytical static solution of the generalized boxed coplanar waveguide," Int. J. Microwave Millimeter-Wave CAE, vol. 4, no. 2, pp. 163-174, Apr. 1994.

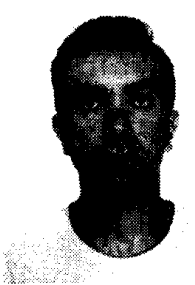

Gabriel Cano received the Lcdo. (M.Sc.) degree in 1988 and the Doctor (Ph.D.) degree in 1993, both in physics from the University of Seville (Spain).

He joined the Microwaves Group (Electronics and Electromagnetics Department) with a grant from the Spanish Government and presently he is assistant professor in the Applied Physics Department of the University of Seville. He focuses his interest on the analysis of planar transmission lines and microwave circuit design.

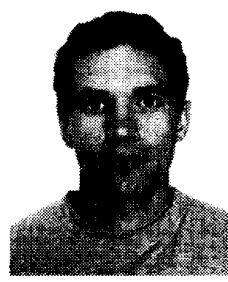

Francisco Mesa received the Lcdo. (M.Sc.) degree in June 1989 and the Doctor (Ph.D.) degree in Dec. 1991, both in physics, from the University of Seville (Spain).

He joined the Microwaves Group (Electronics and Electromagnetics Department) with a grant from the Spanish Government and presently he is assistant professor in the Applied Physics Department of the University of Seville. His research interest focuses on electromagnetic propagation/radiation in planar lines with general anisotropic materials.

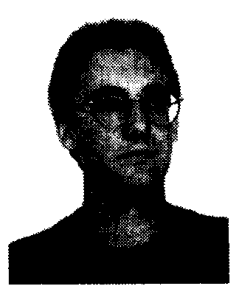

Francisco Medina received the Licenciado degree (with honors) in Sept. 1983 and the Doctor degree in 1987 , both in physics, from the University of Seville, Spain. He spent the 1986-87 academic year at the Laboratoire de Microondes de l'ENSEEIHT (Toulouse, France) with a scholarship of MECMRT.

He was assistant professor at the Department of Electronics and Electromagnetics, University of Seville, during 1985-1989. Since 1990 he has been an associate professor of electricity and magnetism at the same institution. His research deals mainly with analytical and numerical methods for planar structures and analysis and applications of multiconductor systems.

Dr. Medina was member of the Technical Program Committee of the 23rd European Microwave Conference held in Madrid, Spain in Sept. 1993.

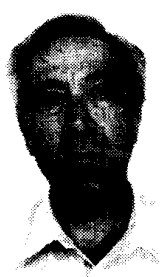

Manuel Horno (M'75) received the Licenciado degree in physics in June 1969, and the Doctor degree in physics in Jan. 1972, both from the University of Seville, Spain.

Since Oct. 1969 he has been with the Department of Electronics and Electromagnetism at the University of Seville, where he became an assistant professor in 1970, associate professor in 1975, and professor in 1986. He is a member of Electromagnetism Academy of M.I.T, (Cambridge, MA). His main fields of interest include boundary value problems in electromagnetic theory, wave propagation through anisotropic media, and microwave integrated circuits. He is presently engaged in the analysis of planar transmission lines embedded in complex materials, multiconductor transmission lines, and printed antennas. 